\title{
Revisiting Microstructure Sensor Responses with Implications for Double-Diffusive Fluxes
}

\author{
TOBIAS SOMMER \\ Eawag, Surface Waters Research and Management, Kastanienbaum, and Institute of Biogeochemistry \\ and Pollutant Dynamics, ETH Zürich, Zurich, Switzerland \\ JEFFREY R. CARPENTER \\ Eawag, Surface Waters Research and Management, Kastanienbaum, Switzerland, and Department of Geology \\ and Geophysics, Yale University, New Haven, Connecticut \\ MARTIN SCHMID \\ Eawag, Surface Waters Research and Management, Kastanienbaum, Switzerland \\ ROLF G. LUECK \\ Rockland Scientific International Inc., Victoria, British Columbia, Canada \\ Alfred WÜEST \\ Eawag, Surface Waters Research and Management, Kastanienbaum, Institute of Biogeochemistry \\ and Pollutant Dynamics, ETH Zürich, Zurich, and Margaretha Kamprad Chair of Environmental Science \\ and Limnology, Physics of Aquatic Systems Laboratory, ENAC, EPFL, Lausanne, Switzerland
}

(Manuscript received 20 December 2012, in final form 5 March 2013)

\begin{abstract}
Thin high-gradient interfaces that occur naturally within double-diffusive staircases are used to estimate the response characteristics of temperature and conductivity microstructure sensors. The knowledge of these responses is essential for resolving small-scale turbulence in natural water bodies and for determining doublediffusive fluxes of heat and salt. Here, the authors derive microstructure sensor responses from observed differences in the statistical distributions of interface thicknesses at various profiling speeds in Lake Kivu (central Africa). In contrast to the standard approach for determining sensor responses, this method is independent of any knowledge of the true in situ temperature and salinity structure. Assuming double-pole frequency response functions, the time constants for the Sea-Bird Electronics SBE-7 conductivity sensor and the Rockland Scientific International FP07 thermistor are estimated to be 2.2 and $10 \mathrm{~ms}$, respectively. In contrast to previous assumptions, the frequency response for the SBE-7 is found to be substantial and dominates the wavenumber response for profiling speeds larger than $0.19 \mathrm{~m} \mathrm{~s}^{-1}$.
\end{abstract}

\section{Introduction}

Turbulent mixing in stratified water bodies generates small-scale structures on the order of millimeters or less. For in situ turbulence studies, those naturally occurring fluctuations need to be resolved as perfectly as possible

Corresponding author address: Tobias Sommer, Eawag, Seestrasse 79, CH-6047 Kastanienbaum, Switzerland.

E-mail: tobias.sommer@eawag.ch
(Gregg 1999). However, currently available sensors usually reach their measurement limits before a complete resolution of the finest turbulent scales is possible. To extract the maximum variance of those turbulent fluctuations, measured signals are routinely corrected for their sensor response properties.

In general, sensors have a frequency (temporal) response and a wavenumber (spatial) response. The frequency response is a consequence of temporal delays of the sensor to the environmental signal. The wavenumber 

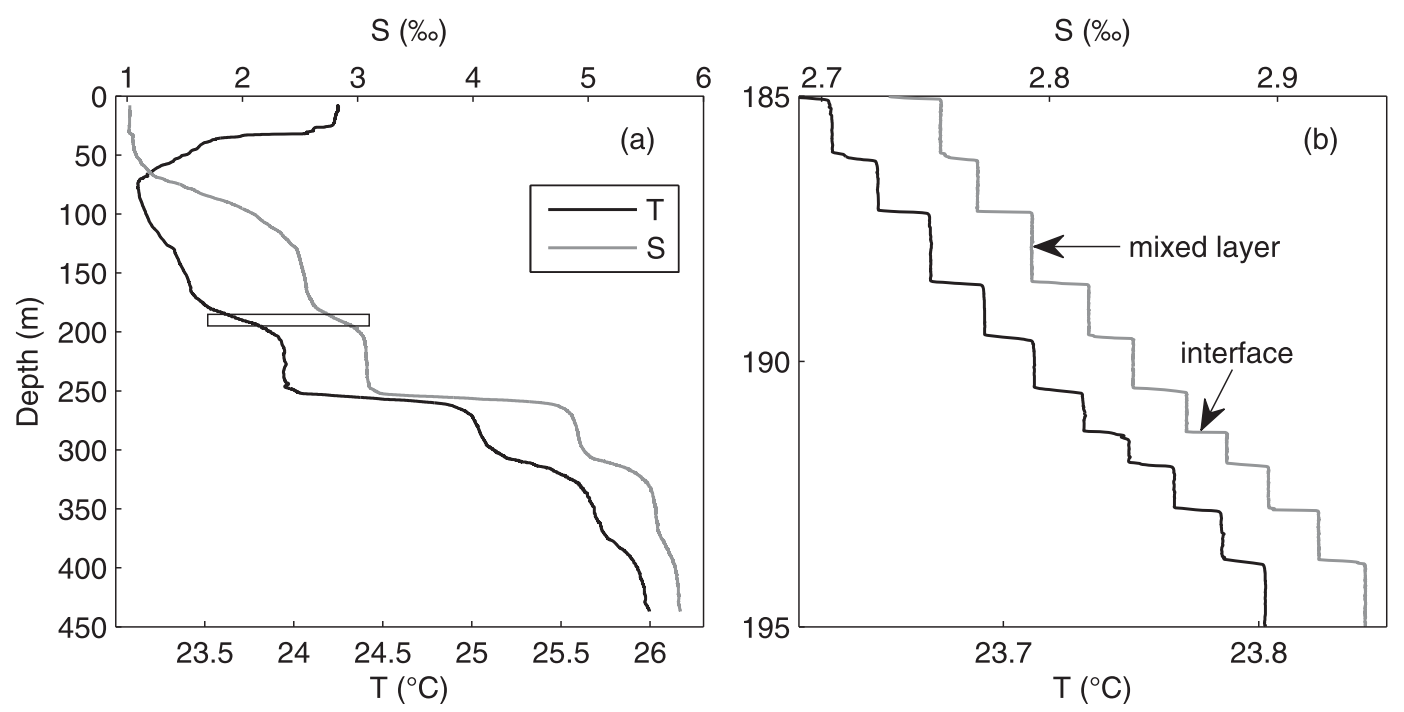

FIG. 1. (a) Typical Lake Kivu profiles of temperature and salinity. (b) Double-diffusive staircases within the 10-m-long section marked by the rectangle in (a).

response is a consequence of the finite size of the sensor, rendering it unable to resolve finer-scale features. It averages the signal over a certain volume determined by the spatial characteristics of the sensor.

Sensor responses are usually determined by comparing the "true" signal, to which the sensor is exposed, with the sensor's measurement output. The response characteristics are modeled by a transfer function that is given by the ratio of the output to the input in the Laplace domain (Dorf and Bishop 2010). However, in practice, the true signal is usually not well known and has either to be estimated (Head 1983) or measured with another sensor with higher spatial resolution than the one being tested (Nash et al. 1999; Schmitt et al. 2005). This is a potential source of error, in particular if the sensor being tested is already very fast.

Here, we take a different approach to finding sensor frequency responses that does not depend on knowledge of the true input. Instead, we take advantage of the fact that, if the sensor shows a frequency response, the measured signal of a moving sensor is speed dependent. Fast profiling will result in increased smoothing of the measured signal, as structures of a given spatial scale are detected over a short time scale. The shorter the time scale, the more the signal is affected by the frequency response. The frequency response can thus be found by correcting the signals, measured at different profiling speeds, such that they become identical. At the same time the true signal, initially unknown, is approximated with increasing accuracy. This approach assumes that either the true signal itself or the distribution of a certain characteristic of the true signal is stationary.
Lake Kivu offers an attractive natural environment for examining the frequency response characteristics of temperature $T\left({ }^{\circ} \mathrm{C}\right)$ and electrical conductivity $C\left(\mu \mathrm{S} \mathrm{cm}^{-1}\right)$ microstructure sensors. Depth profiles of the lake show more than 300 homogeneous layers separated by thin high-gradient interfaces, a phenomenon produced by double diffusion of the diffusive type (Neal et al. 1969; Newman 1976; Schmitt 1994; Kelley et al. 2003; Timmermans et al. 2008). Figure 1a shows background profiles of $T$ and salinity $S$ in Lake Kivu and the closeup in Fig. 1b illustrates an example of the staircase structure consisting of a series of stable interfaces sandwiched between convectively mixed layers. Double-diffusive interfaces have previously been used to study the responses of CTD sensors (Schmitt et al. 2005; Johnson et al. 2007). The goal of these studies was to match the $T$ and $C$ signals during postprocessing in order to avoid "salinity spiking," which would result in "density spiking." However, those methods are not applicable to very fast microstructure sensors: the method by Schmitt et al. (2005) requires an assumption for the true signal, which for very fast microstructure sensors would strongly affect the deduced sensor response. The method by Johnson et al. (2007) is based on reconstructing the true signal from the measured signal (Fofonoff et al. 1974; Fozdar et al. 1985). This requires the removal of high-frequency instrument noise by smoothing the measured signal. However, for fast microstructure sensors, those high frequencies contain essential information about the response and thus smoothing is problematic.

Our method, in contrast, only assumes that doublediffusive interface thickness distributions are stationary over the time of our measurement. We believe that this 
is a justified assumption because the background profiles of $T$ and $S$ in Lake Kivu (Fig. 1a), on top of which the staircases are formed, are persistent both temporally and spatially. The variation between $T$ and $C$ profiles measured in 2004 by Schmid et al. (2010) and in 2010 and 2011 by the current team is largest near the lake bottom (excluding the top $60 \mathrm{~m}$, which are affected by seasonal variation). At $450 \mathrm{~m}$ the ranges for $T$ and $C$ are $25.99 \pm$ $0.05^{\circ} \mathrm{C}$ and $5775 \pm 25 \mu \mathrm{S} \mathrm{cm}^{-1}$, respectively, corresponding to $0.4 \%$ and $0.9 \%$. Thus, the statistical properties of the staircases are assumed to evolve slowly and to be independent of profiling location in the open water. Here, we use this quasi stationarity to explore the frequency response of two microstructure sensors by measuring interface thicknesses at three different profiling speeds. Our microstructure sensors are the FP07 thermistor [Rockland Scientific International (RSI), Canada and General Electric (GE) Energy, United States], often used for the measurement of $T$, and the Sea-Bird SBE-7 microconductivity sensor (Sea-Bird Electronics, United States) for the measurement of $C$. Differences in the distributions measured at those speeds are assumed to be caused by the sensor frequency response. The frequency response is then estimated by correcting individual interface thicknesses such that the corrected distributions collapse to a single distribution. In particular, we show (i) that the SBE-7 sensor (in addition to the FP07 sensor) has a frequency response and (ii) that the deduced corrections result in improved estimates of vertical heat and salt fluxes as well as density flux ratios through double-diffusive staircases.

The paper is structured as follows: we first describe the vertical microstructure profiler (section $2 \mathrm{a}$ ), introduce the dataset measured at Lake Kivu (section 2b), and outline the method for interface detection (section 2c). We then present our assumptions on the functional forms of the responses (section 3a). Based on those responses we introduce the model approach for correcting interface thicknesses (section 3b). In section 4, we show that measured interface thicknesses depend on profiling speed and how we remove this dependency by correcting for the frequency responses. In section 5 , the estimated responses are discussed with respect to findings by other researchers, and in section 6 , we investigate the effect of the sensor response correction on the estimates of physical parameters in Lake Kivu, such as vertical fluxes of heat and salt and the ratio of $T$ to $S$ interface thicknesses. We finally summarize our observations in section 7 .

\section{Methods}

\section{a. Vertical microstructure profiler}

Profiles of $T$ and $C$ microstructure are measured with a loosely tethered, and thus quasi-free falling, vertical microstructure profiler (VMP; Fig. 2a) manufactured by RSI. The instrument is $163 \mathrm{~cm}$ long and weighs $340 \mathrm{~N}$ in air.

During profiling, the tether is released from a motordriven winch slightly faster than the VMP descends, ensuring that it is not perturbed by any ship motions. The VMP descent is stabilized by two brushes, located at the rear, and a flotation collar. Three DC-response M4002 accelerometers (Precision Measurements Systems, United States) record pitch, roll, and vibrations. Depth (m) is calculated by multiplying pressure (bar) by $10.213 \mathrm{~m} \mathrm{bar}^{-1}$, which accounts well for the density of Lake Kivu (Schmid et al. 2005) and has a maximum absolute error of $0.36 \mathrm{~m}$ at $250-\mathrm{m}$ depth. The VMP profiling speed is computed from the low-pass-filtered rate of change of pressure.

The VMP is optimized for slow profiling down to $0.1 \mathrm{~m} \mathrm{~s}^{-1}$ and thus differs from, for example, the TurboMAP profiler in Wolk et al. (2002). For slow profiling, the electronics were modified (as described below) and a flotation collar is mounted. The target profiling speed is then tuned by adding lead weights. We profile at three different speeds: $W=0.19 \mathrm{~m} \mathrm{~s}^{-1}$ (slow), $0.38 \mathrm{~m} \mathrm{~s}^{-1}$ (medium), and $0.89 \mathrm{~m} \mathrm{~s}^{-1}$ (fast). The cast properties for profiling at different speeds are summarized in Table 1.

Three pairs of microstructure sensors (Figs. 2b,c) are mounted at the nose of the instrument where they are exposed to the undisturbed water. As sensors, we use two fast FP07 (MicT) thermistors, two fast SBE-7 (MicC) conductivity microsensors (Fig. 1d), and two SPM-38shear probes (RSI, Canada) sampled at a frequency $f_{S}=512 \mathrm{~Hz}$.

Microstructure sensors are very sensitive but lack long-term stability. The temporal drifts of the signals are removed by calibrating the microstructure signals against the stable but slower $C$ and $T$ signals from a SeaBird SBE-4C and SBE-3F, respectively, sampled at $64 \mathrm{~Hz}$, which are also mounted on the VMP (Fig. 2a). The calibration is done by linearly adjusting the microstructure signals to these slow CTD sensors over the depth interval from $100 \mathrm{~m}$ to the maximum depth of a profile. The fit error relative to the maximum variation of $T$ and $C$ over the entire profile is smaller than $1.7 \%$ for both $T$ and $C$. The flow through the glass cylinder of the SBE-4C was enhanced by a submersible pump (SBE-5T) for most profiles. During the campaign in 2010, the glass cell of the SBE-4C was cracked, which resulted in erroneous background data. For these profiles, the MicC signal is mapped on an average background profile measured in 2011. This is unproblematic because time as well as horizontal variations of $C$ from 2004 to 2011 are smaller than $1 \%$. 

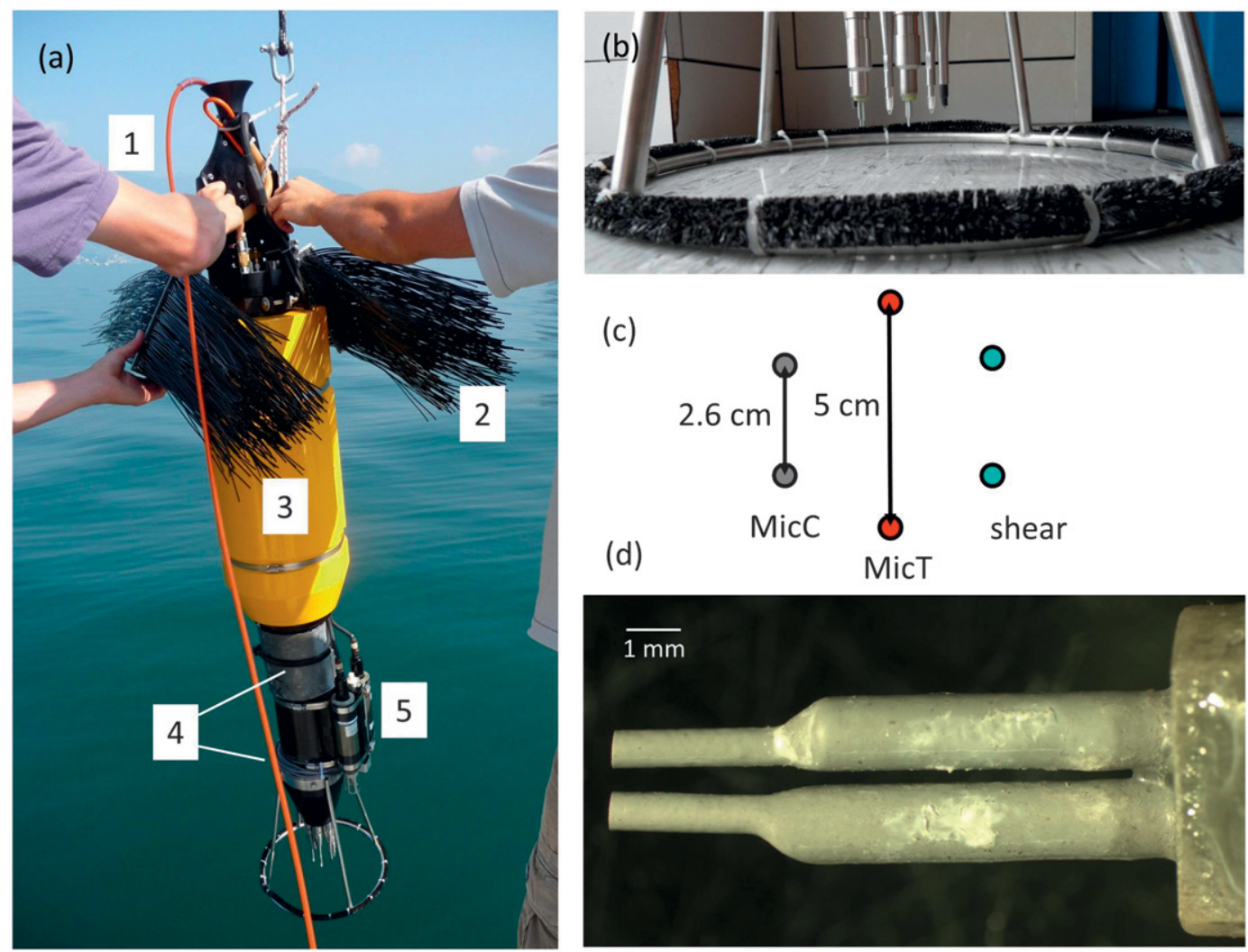

FIG. 2. (a) VMP with 1) orange tether cable, 2) stabilizing brushes, 3) yellow flotation collar, 4) lead, and 5) SeaBird $C$ and $T$ sensors. (b) Six microstructure sensors mounted at the instrument nose and protected by a metal frame covered by black brushes. (c) Microstructure sensor arrangement when facing the instrument nose. (d) Conductivity sensor SBE-7. The electrodes are situated on the tips of the two needles. The distance between the tips defines the parameter $2 a$ of the response function by Hill and Woods (1988). Note the salt precipitations in white after deployment in Lake Kivu.

To reduce quantization noise in the high-frequency range, a preemphasis and deconvolution method (Mudge and Lueck 1994) is applied to both microstructure sensors. The signal conditioning board of the internal electronics is optimized for slow profiling by increasing the preemphasis gain of the MicT about ninefold compared to that used for normal profiling speeds $\left(W=\sim 1 \mathrm{~m} \mathrm{~s}^{-1}\right)$. The gain of the MicC is not enhanced because the analog noise is already above the quantization noise and thus amplifying the signal at high frequencies would only cause amplification of analog noise. The standard deviation of the noise signal of the two MicC sensors is

TABLE 1. VMP operation and data acquisition. Values given for speed, pitch, and roll were measured in the depth range of detected interfaces.

\begin{tabular}{lcccc}
\hline & Unit & Slow & Medium & Fast \\
\hline Average profiling speed & $\mathrm{m} \mathrm{s}^{-1}$ & 0.19 & 0.38 & 0.89 \\
Profiling speed (min/max) & $\mathrm{m} \mathrm{s}^{-1}$ & $0.10 / 0.28$ & $0.30 / 0.42$ & $0.70 / 0.96$ \\
Profiling speed (10th/90th percentiles) & $\mathrm{m} \mathrm{s}^{-1}$ & $0.14 / 0.24$ & $0.38 / 0.39$ & 21 \\
Time for 485 m downcast at average speed & $\mathrm{min}$ & 43 & 5897 \\
Total number of detected interfaces & - & 2199 & 58 & 2141 \\
Percentage of total interfaces & $\%$ & 21 & yes & 5.3 \\
Floating collar mounted? & - & 2.7 & $-1.9 /-1.5$ & no \\
Lead added & $\mathrm{kg}$ & $-3.3 /-0.5$ & $0.2 / 0.5$ & $-2.8 /-1.8$ \\
Pitch (10th/90th percentiles) & $\circ$ & $-2.2 / 3.4$ & $-2.5 /-0.2$ \\
Roll (10th/90th percentiles) & $\circ$ & 0.4 & 0.7 \\
Vertical distance between measurement points & $\mathrm{mm}$ & & 1.7 \\
\hline
\end{tabular}


0.12 and $0.06 \mu \mathrm{S} \mathrm{cm}^{-1}$ as well as $0.012 \mathrm{mK}$ for both MicT sensors. Noise measurements were conducted in a laboratory tank under quiet conditions. The two MicC sensors have different noise levels because their transformers have different material in their cores.

The spatial resolution depends on sampling frequency, profiling speed, and the wavenumber and frequency responses of the sensors and internal electronics. Each aspect is discussed in detail in the following sections.

\section{b. Lake Kivu dataset}

Measurements were carried out in Lake Kivu (surface area of $2300 \mathrm{~km}^{2}$, maximum depth of $485 \mathrm{~m}$, and volume of $550 \mathrm{~km}^{3}$ ), located at the border between Rwanda and the Democratic Republic of the Congo (Schmid et al. 2005; Descy et al. 2012). During two field campaigns in 2010 and 2011 we measured 225 profiles covering a total profiling length of approximately $55 \mathrm{~km}$, containing about 10000 double-diffusive steps. Each step was simultaneously measured by four microstructure sensors, two MicT and two MicC. For a more detailed description of the lake and its double-diffusive staircases we refer to Newman (1976), Schmid et al. (2010), and Sommer et al. (2013, manuscript submitted to J. Geophys. Res.).

\section{c. Interface detection}

To manage the enormous dataset, we developed an algorithm for automatic evaluation of the measured profiles. The algorithm detects double-diffusive interfaces and mixed layers in the measured profiles and extracts interface thicknesses, mixed-layer thicknesses, step sizes of $T$ and $C$ between mixed layers, and several more parameters. In this work, we focus on interface thicknesses defined by $h_{T}=\left.\Delta T(\partial T / \partial z)^{-1}\right|_{z=z_{0}}$ for temperature and $h_{C}=\left.\Delta C(\partial C / \partial z)^{-1}\right|_{z=z_{0}}$ for conductivity. Here $\Delta T(\mathrm{~K})$ and $\Delta C\left(\mu \mathrm{S} \mathrm{cm}^{-1}\right)$ are the $T$ and $C$ steps across the interface (Fig. 3), respectively, $z(\mathrm{~m})$ is depth (positive downward), and $z_{0}(\mathrm{~m})$ is the depth of the interface center. The same definition holds for the salinity interface thickness $h_{S}$.

The detection algorithm is based on four mandatory conditions:

- Condition (i) - the interfacial gradient is large compared to the background gradient.

- Condition (ii) - the gradients of the two adjacent mixed layers are small compared to the background gradient.

- Condition (iii) —all four microstructure sensors detect the same interface based on arguments (i) and (ii).

- Condition (iv) - profiles of $T$ and $C$ are close to linear in their interface core. More $C$ than $T$ interfaces are
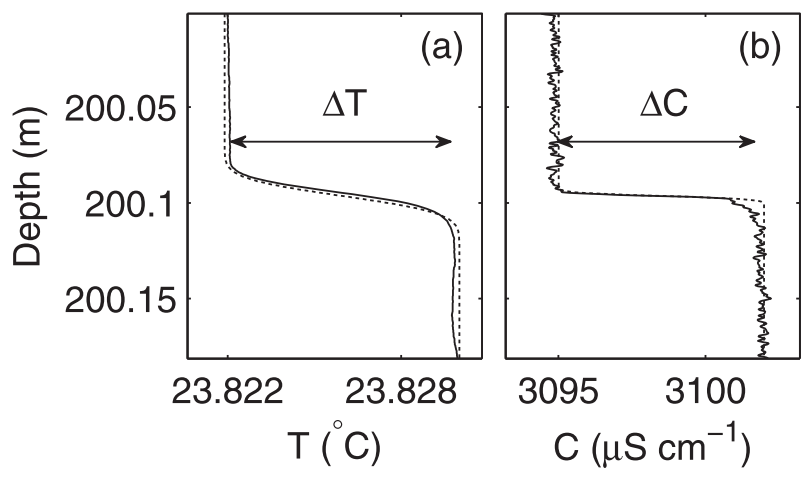

FIG. 3. Example of a 20 -cm-long profile section with simultaneously measured and well-resolved (a) temperature and (b) conductivity interfaces (solid lines). The error function approximation is depicted by the dotted lines. Note the delay of the conductivity signal at the lower edge of the interface, which is an indication for the temporal response.

rejected by this condition because the ratio of noise to step size for the MicC is larger than for the MicT and because $S$ diffuses more slowly than $T$ and therefore disturbances in the $C$ interfaces persist over longer times.

Each of the conditions is described in detail in appendix A. In total, $17619 T$ interfaces and $9489 C$ interfaces passed all four conditions and are evaluated in the following section.

\section{Sensor response formulation}

Measured interfaces are always smoothed out by the sensor responses and thus appear thicker than the true in situ interfaces. If the sensor has a temporal response only then this response is fully described by the impulse response function $h(t)$ with $t(\mathrm{~s})$ being time, which is the output signal for a delta function input. In practice, the Fourier transform of $h(t)$, which we call frequency response $H(f)$, is often used to describe amplitude and phase changes as a function of frequency $f(\mathrm{~Hz})$ (Dorf and Bishop 2010). In the spatial domain, we use the identical notation for the spatial impulse response $h(z)$ and the wavenumber response $H(k)$ with $k$ [cycles per meter (cpm)] being the wavenumber. For a given input, such as a model interface, we simulate the output (representing the measured signal) by a convolution of the input with $h(z)$ and/or $h(t)$. This way, we establish a relation between the thickness of the input interface and the output interface, and we use this relation to infer true interface thicknesses in Lake Kivu. In the following we introduce our assumptions for the sensor responses and then describe our method for correcting measured interface thicknesses. 


\section{a. Sensor responses}

We distinguish between a wavenumber response and a frequency response, and we explain each contribution briefly for both sensor types.

\section{1) WAVENUMBer RESPONSE, MicT}

The FP07 thermoprobe is a tiny glass-coated thermistor bead with a diameter of approximately $180 \mu \mathrm{m}$. Because the smallest $T$ scales of interest are about $1 \mathrm{~cm}$, the MicT wavenumber response is not a limiting factor, and it is omitted.

\section{2) WAVEnumber Response, MicC}

The SBE-7 conductivity sensor measures the conductivity of the fluid by applying a constant-amplitude sinusoidal voltage difference between its electrodes (Fig. 2d). This voltage difference drives a current through the fluid and the amplitude of the current is proportional to $C$. However, the current path lines spread apart in the volume between and around the electrodes and the measured $C$ represents an average over this volume. The smoothing of the spatial $C$ fluctuations resulting from this averaging is described by a wavenumber response. We use the Hill and Woods (1988) form $H(k)=$ $\exp (-2 \pi k a)$ with $a=0.23 \mathrm{~mm}$ being half the distance between electrodes. The corresponding impulse response function is $h(z)=Q a\left(z^{2}+a^{2}\right)^{-1}$, where $Q$ is a normalization constant.

\section{3) Frequency Response, Mict}

The frequency response of the MicT depends on how fast heat is transported into the viscous boundary layer and diffused through the diffusive boundary layer as well as the glass coating to the thermistor. The frequency response characteristics of the FP07 are not known exactly and different findings are discussed in section 5a. We follow Gregg and Meagher (1980) and use their double-pole frequency response function $H_{\mathrm{dp}}(f)=(1+$ $\left.j 2 \pi f \tau_{\mathrm{dp}}\right)^{-2}$, where $j$ is the imaginary unit and $\tau_{\mathrm{dp}}(\mathrm{s})$ is the time constant specific for a double-pole response. Here, $\tau_{\mathrm{dp}}$ is the single parameter that we use to characterize the temporal response, and one goal of this study is to determine this parameter. The time constant $\tau_{\mathrm{dp}}$ was reported to depend on profiling speed $W$ because the water boundary layer around the sensor becomes thinner for higher $W$ and thus heat can diffuse faster to the sensor. For a velocity scaling of the form

$$
\tau_{\mathrm{dp}}=\tau_{0}\left(W / W_{0}\right)^{\gamma}
$$

with $W_{0}=1 \mathrm{~m} \mathrm{~s}^{-1}$ and $\tau_{0}=\tau_{\mathrm{dp}}$ at $W=W_{0}$, the exponents $\gamma=-0.32$ (Gregg and Meagher 1980) and $\gamma=-0.5$
(Vachon and Lueck 1984; Hill 1987) have been proposed. We neglect the velocity dependence on $\tau_{\mathrm{dp}}$ based on the results described in this section. The corresponding impulse response function is $h(t)=\tau_{\mathrm{dp}}^{-2} \exp \left(-t / \tau_{\mathrm{dp}}\right)$ for $t>0 ; h(t)=0$ otherwise. However, instead of convolving with $h(t)$, we filter twice with a first-order lowpass Butterworth filter with a cutoff frequency of $\left(2 \pi \tau_{\mathrm{dp}}\right)^{-1}$, which is less time consuming and yields a better representation of $H_{\mathrm{dp}}(f)$ for a discrete input signal.

\section{4) Frequency Response, MicC}

The MicC sensor is usually considered infinitely fast and thus frequency response corrections are not applied to the $C$ signal (Meagher et al. 1982; Head 1983; Hill and Woods 1988; Washburn et al. 1996; Nash and Moum 2002). However, our observations (section 4a) indicate that the MicC does have a frequency response. Both the delayed flushing of the viscous boundary layer by the flow and the processing of the signal can produce a frequency response and their contributions are discussed in section 5b. For the MicC, we assume the same doublepole frequency response function as for the MicT with variable $\tau_{\mathrm{dp}}$. This is an arbitrary choice but facilitates direct comparison of the time constants.

\section{b. Modeling interface thickness corrections}

The relation between true $\left(h_{\text {true }}\right)$ and observed $\left(h_{\text {meas }}\right)$ interface thicknesses depends on the sensor response with the parameter $\tau_{\mathrm{dp}}$ and on the profiling speed $W$. In the following, we derive six continuous functions $N\left(h_{\text {meas }}, \tau_{\mathrm{dp}}, W\right)$ for this relationship for our two sensors and three profiling speeds. Here, $N$ corresponds to the fraction of $h_{\text {meas }}$, which must be subtracted from $h_{\text {meas }}$ in order to obtain $h_{\text {true: }}$ :

$$
N\left(h_{\text {meas }}, \tau_{\text {dp }}, W\right)=1-h_{\text {true }} / h_{\text {meas }} .
$$

The true thickness of an interface is then calculated (for each sensor and $W$ ) from the measured interface thickness by $h_{\text {true }}=(1-N) h_{\text {meas }}$.

The functions $N$ are derived by simulating the sensor responses for a series of hypothetical model interfaces with thicknesses $h_{\text {true }}^{*}$ and $h_{\text {meas }}^{*}$ before and after the sensor response correction has been applied, respectively. The discrete correction factors $N^{*}\left(h_{\text {meas }}^{*}, \tau_{\mathrm{dp}}, W\right)$ are then calculated for each pair of $\left(h_{\text {meas }}^{*}, h_{\text {true }}^{*}\right)$ from Eq. (2). Finally, continuous functions are fitted to $N^{*}$ to interpolate between the simulated $h_{\text {meas }}^{*}$. As a fit function, we used

$$
\begin{aligned}
N\left(h_{\text {meas }}, \tau_{\mathrm{dp}}, W\right)= & 0.043+A \exp \left[B\left(h_{\text {meas }}+D\right)\right] \\
& +C\left(h_{\text {meas }}+D\right)^{-1}
\end{aligned}
$$


where the dependency on $\tau_{\mathrm{dp}}$ and $W$ is included in the fit parameters $A, B, C$, and $D$. The combination of a constant term, an exponential term, and an $x^{-1}$ term on the right-hand side of Eq. (3) was found after testing various other relations, which did not provide a satisfactory fit to the modeled data. Apart from the purely empirical functional form, Eq. (3) is designed to fulfill three additional conditions: (i) the constant of 0.043 accounts for underestimating the gradient by fitting over the center $50 \%$ of the interface (appendix A), (ii) forcing $B<0$ ensures that the correction decreases for large $h_{\text {meas }}$, and (iii) the fit parameter $D$ in the $x^{-1}$ term allows the fit function to rapidly increase at some $h_{\text {meas }}$, a behavior that was observed for all model runs.

As model $T$ and $C$ interfaces, we use error functions of the form

$$
T(z)=0.5 \Delta T \operatorname{erf}\left[\pi^{1 / 2}\left(z-z_{0}\right) / h_{\text {true }}^{*}\right]
$$

and

$$
C(z)=0.5 \Delta C \operatorname{erf}\left[\pi^{1 / 2}\left(z-z_{0}\right) / h_{\text {true }}^{*}\right],
$$

respectively. We use the error function form because it is a solution of the diffusion equation, and its shape is close to observed interfaces (Fig. 3). The vertical spacing $\Delta z$ of the sampling for the modeled step was chosen as $W\left(4 f_{S}\right)^{-1}$. The oversampling by a factor of 4 ensures that the Nyquist frequency $f_{N}=0.5 f_{S}$ is a few times larger than the half-power frequencies $f_{h}$, defined as $\left|H\left(f_{h}\right)\right|^{2}=$ 0.5 , of the applied digital filters, and thus discretization errors are small. In our case $f_{N}>6 f_{h}$ is satisfied for all filters. We then apply the sensor responses of MicT and MicC to Eqs. (4) and (5), respectively, in order to obtain individual $h_{\text {meas }}^{*}$ for each sensor and profiling speed, as described in the following sections.

\section{1) $\mathrm{MICT}$}

We convert our model step $T(z)$ [Eq. (4)] into a time series step $T(t)$ using $t=z / W$ and then apply the doublepole frequency response $H_{\mathrm{dp}}(f)$ (section 3a). Note that an interface of thickness $h$ is traversed in the time interval $\Delta t=h / W$. The larger $W$ is, the smaller the $\Delta t$ is and the stronger the smoothing of the frequency response function on the time series step is. This effect of the frequency response causes measured interface thicknesses to be profiling speed dependent. After back transformation into the spatial domain, the interface thickness $h_{\text {meas }}^{*}$ of the filtered step is determined with the same method as used for interfaces measured in Lake Kivu (appendix A). This is done for a series of 100 different values of $h_{\text {true }}^{*}$, which are logarithmically spaced between $0.1 \mathrm{~mm}$ and $1 \mathrm{~m}$. The logarithmic scale ensures tight spacing at small interface thicknesses where the correction is most important.

\section{2) $\mathrm{MICC}$}

For the MicC, we follow the same procedure as for the MicT but additionally account for the wavenumber response described in Hill and Woods (1988) before transforming the spatial model step into the time domain.

\section{Results}

\section{a. Measured interface thicknesses}

In Figs. $4 \mathrm{a}$ and $4 \mathrm{~b}$, the normalized histograms of measured $h_{T}$ and $h_{C}$ are shown for different profiling speeds with MicT results on the left and MicC on the right. No sensor response corrections are applied. Note that the distributions measured by the two MicT (Fig. 4a) and the two MicC (Fig. 4b) sensors are practically identical to each other. This is not necessarily expected for the MicT, as Nash and Moum (1999) as well as Gregg and Meagher (1980) found that individual sensors may have different response characteristics. Also note that the different noise levels of the MicCs do not affect the $h_{C}$ distributions. The algorithm for calculating interface thicknesses needs at least two points in the central 50\% of the interface for fitting (appendix A), and thus interfaces containing fewer than four points are not detected. The thresholds corresponding to these conditions are indicated by vertical lines in Fig. 4 for each of the three profiling speeds. They are determined solely by the sampling frequency and the algorithm. Interfaces thinner than these thresholds are ignored by the algorithm and would result in a steep cutoff in the measured distributions. Since this is not seen in Figs. $4 a$ and $4 b$, we conclude that the distributions are unaffected by the thresholds, which indicates that a sufficiently fast sampling rate is used. The distribution of $h_{C}$ using fast profiling, however, is very close to its corresponding threshold (Fig. 4b).

For both sensors, the histograms of the thin interfaces exhibit a clear dependence on the profiling speed. We believe that this is caused by the frequency responses of the sensors. This is expected for the MicT but a surprise for the $\mathrm{MicC}$, as those sensors are commonly assumed to be infinitely fast (Meagher et al. 1982; Head 1983; Hill and Woods 1988; Washburn et al. 1996; Nash and Moum 2002). After applying the appropriate corrections for the interface thicknesses the histograms should be identical for all three profiling speeds.

\section{b. Corrected interface thicknesses}

In Fig. $4 \mathrm{c}, h_{T}$ is corrected as described in section $3 \mathrm{~b}$ using $H_{\mathrm{dp}}(f)$ with the $\tau_{\mathrm{dp}}$ of Vachon and Lueck (1984). 
MicT
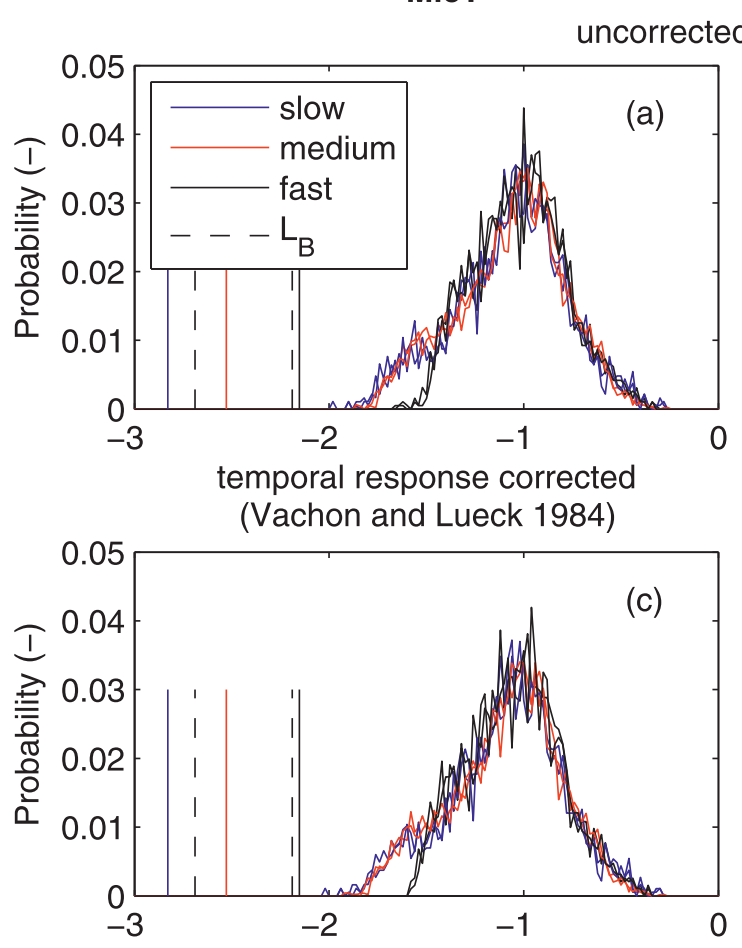

MicC

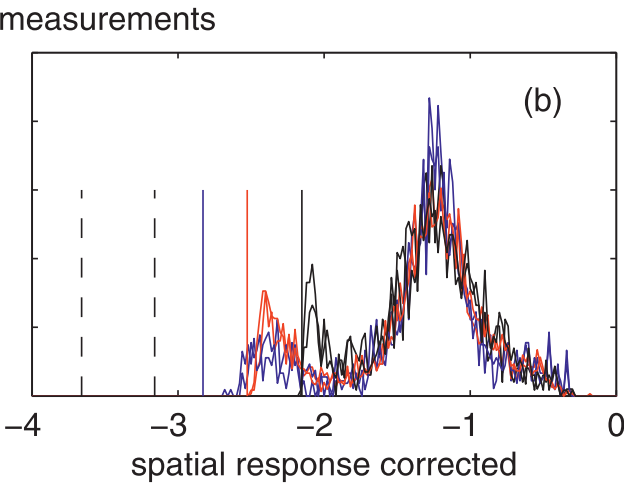

(Hill and Woods 1988)

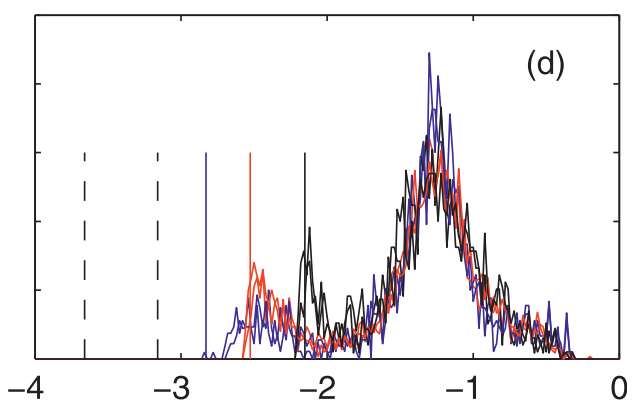

inferred corrections
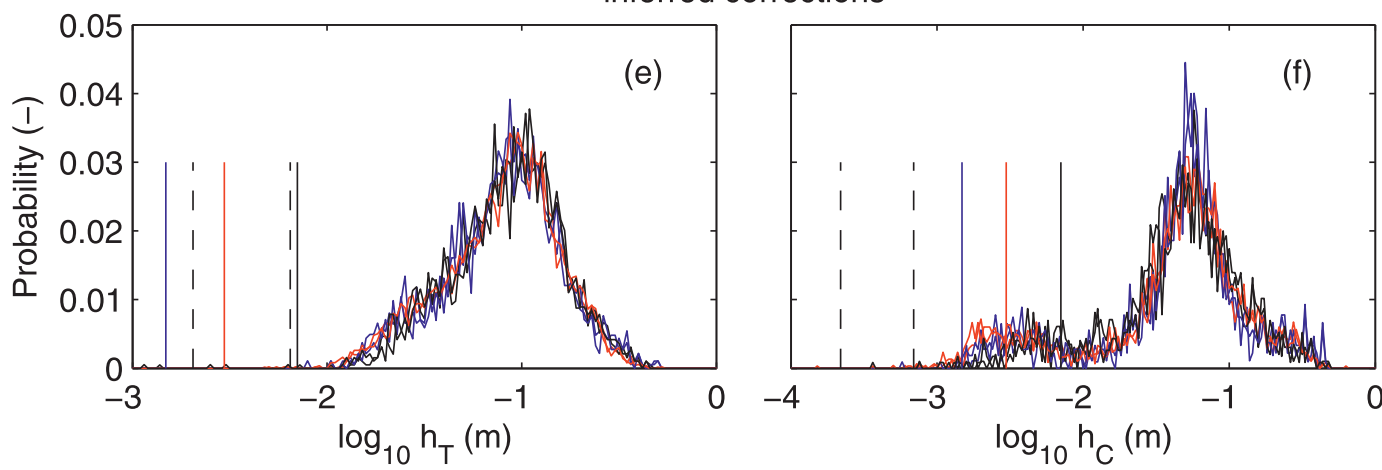

FIG. 4. Effect of microstructure sensor responses on interface thickness distributions for different profiling speeds. All histograms are based on logarithmic bin sizes of 0.02 and are normalized by the total number of data points. The two almost identical lines for each profiling speed represent the individual measurements of the two sensors. (a),(b) Measured distributions of $h_{T}$ and $h_{C}$, (c) $h_{T}$ after correcting according to the frequency response of Vachon and Lueck (1984), (d) $h_{C}$ after correcting according to the wavenumber response of Hill and Woods (1988), and (e),(f) modified corrections eliminating most of the profiling speed dependence. Vertical solid lines indicate the smallest measurable interface thickness (details in text). The two vertical dashed lines are Batchelor scales for dissipation rates of $\varepsilon=$ (left) $10^{-11}$ and (right) $10^{-9} \mathrm{~W} \mathrm{~kg}^{-1}$, corresponding to the range of $\varepsilon$ observed in the mixed layers (Sommer et al. 2013, manuscript submitted to J. Geophys. Res.). The number of interfaces measured by sensors 1 and 2 are $(1865,1828),(5069,4993)$, and $(1941,1907)$ for slow, medium, and fast profiling, respectively. For $C$, these numbers are $(899,1075),(2370,3002)$, and $(985,1149)$.

They assume a velocity-dependent time constant $\tau_{\mathrm{dp}}=$ $\tau_{0}\left(W / W_{0}\right)^{-0.5}$ with $\tau_{0}=4.1 \mathrm{~ms}$ and $W_{0}=1 \mathrm{~m} \mathrm{~s}^{-1}$. In Fig. $4 \mathrm{~d}, h_{C}$ is corrected for the wavenumber response $H(k)$ of Hill and Woods (1988) with $a=0.23 \mathrm{~mm}$. After correction, both histograms still depend on profiling speed, indicating that the response characteristics of MicT and MicC are not yet captured adequately. This is obvious for the $\mathrm{MicC}$ because a wavenumber response alone does not introduce a profiling speed dependency of the measured data, and thus the corresponding 
correction cannot remove it. However, note that both corrections slightly decrease the thickness of the thinnest interfaces.

In our final correction (Figs. 4e,f), we keep the MicC wavenumber response of Fig. $4 \mathrm{~d}$ constant but include frequency responses $H_{\mathrm{dp}}(f)$ with variable $\tau_{\mathrm{dp}}$ for both sensor types. We then adjust $\tau_{\mathrm{dp}}$ separately for MicC and MicT to optimize the collapse of the corrected distributions for the three profiling speeds. We find that time constants $\tau_{\mathrm{dp}}$ of 10 and $2.2 \mathrm{~ms}$ for MicT and MicC, respectively, provide the best collapse based on visual inspection. Assuming $\tau_{\mathrm{dp}}$ to be speed dependent (e.g., as in Vachon and Lueck 1984) results in either an insufficient correction for fast profiling or a large number of negative interface thicknesses for slow profiling, which can be understood by the following example. If we assumed a speed-dependent time constant but still required that for slow profiling the time constant $\tau_{\mathrm{dp} \text {,slow }}$ is equal to the speed-independent time constant $\tau_{\mathrm{dp}}$ from above, then $\tau_{\mathrm{dp}, \text { fast }}<\tau_{\mathrm{dp}}$ for fast profiling [Eq. (1), assuming $\gamma<0$ ]. In that case, the data would not collapse because the interfaces measured at fast profiling are not corrected enough. To achieve a collapse, both $\tau_{\mathrm{dp} \text {,slow }}$ and $\tau_{\mathrm{dp}, \text { fast }}$ must be multiplied by a constant factor $>0$. But then the interfaces measured at slow profiling speed would be corrected more than without the velocity scaling. Because some corrected interface thicknesses are already close to zero without the velocity scaling (Figs. 4e,f), an increase of $\tau_{\text {dp,slow }}$ would cause them to become negative. Therefore, we use a time constant $\tau_{\mathrm{dp}}$ that does not depend on profiling speed.

Even without velocity scaling, the corrections applied in Figs. $4 \mathrm{e}$ and $4 \mathrm{f}$ result in few negative interface thicknesses, namely 16 for $T$ (out of 17619) and 9 for $C$ (out of 9489). These are possibly caused by a slightly wrong response function shape or an overestimation of the interfacial gradients resulting from instrument noise or signal discretization. The few negative interface thicknesses are ignored. Note the proximity of the thinnest $T$ and $C$ interface thicknesses to the Batchelor scales inferred from the approximate dissipation rates measured in the mixed layers.

The corrected histogram of $h_{C}$ (Fig. 4f) shows two peaks, a main peak at approximately $6 \mathrm{~cm}$ and a small peak at approximately $3 \mathrm{~mm}$. At the moment we are unsure whether the small peak is a physical feature that is characteristic of the $C$ interfaces or whether it is an artifact of the measurements, but we plan to address this question in a future publication by comparing our measurements to direct numerical simulation.

Figure 5 shows the correction factor $N$ for our final correction and the fit parameters of Eq. (3) are given in Table 2. For individual sensors, $N$ increases with $W$,

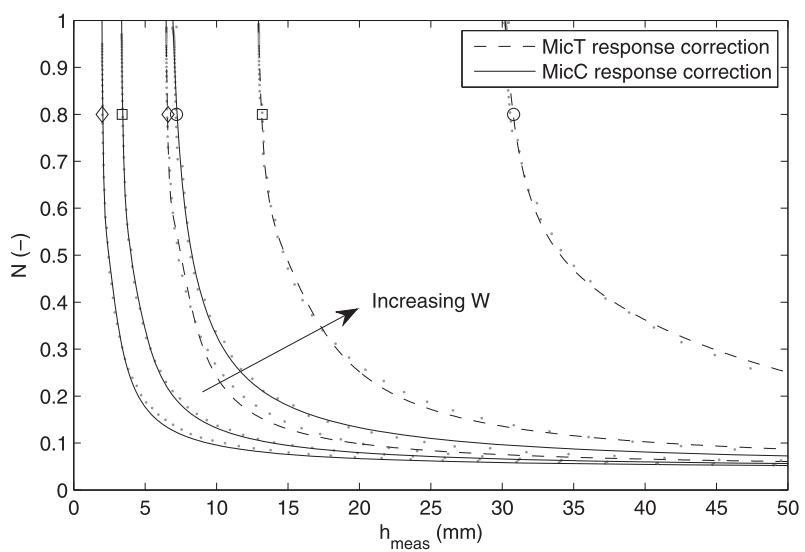

FIG. 5. Interface thickness correction $N$ for measured interface thicknesses $h_{T}$ and $h_{C}$. Points are the modeled data and lines represent fit functions. The fit parameters $A, B, C$, and $D$ are given in Table 2. The plot is restricted to measured thicknesses of less than $50 \mathrm{~mm}$. The sensor responses are profiling speed dependent and the three lines for each sensor correspond to 0.19 (diamonds), 0.38 (squares), and $0.89 \mathrm{~m} \mathrm{~s}^{-1}$ (circles) with the arrow pointing toward increasing $W$.

indicating that faster profiling requires a larger correction. Note that for interfaces thicker than approximately $5 \mathrm{~cm}$ (Fig. 4), the correction is less than $10 \%(N<0.1)$ except for MicT and fast profiling. The value of $h_{\text {meas }}$ for which $N \rightarrow 1$, indicates the smallest directly measurable interface thickness for a certain speed and sensor. For slow profiling this thickness is $6.5 \mathrm{~mm}$ for the MicT and $2 \mathrm{~mm}$ for the MicC. Note that for the MicC a few such interfaces were actually measured (Fig. 4b), which means that their true thickness was close to zero. This becomes an important issue in section 6 where we estimate the molecular fluxes through interfaces.

\section{Discussion of the sensor responses}

In section $4 \mathrm{~b}$ we removed the profiling speed dependence of measured $T$ and $C$ interface thickness estimates by correcting for a double-pole frequency response with the time constants $\tau_{\mathrm{dp}}=10$ and $2.2 \mathrm{~ms}$, respectively. We now discuss these time constants with respect to the findings of other researchers.

TABLE 2. Parameters of Eq. (3) used for interface thickness correction.

\begin{tabular}{rcrrrr}
\hline & \multicolumn{1}{c}{ Speed $\left(\mathrm{m} \mathrm{s}^{-1}\right)$} & $A$ & $B\left(10^{-2} \mathrm{~m}^{-1}\right)$ & $C\left(10^{4} \mathrm{~m}^{-1}\right)$ & $D\left(10^{3} \mathrm{~m}\right)$ \\
\hline$h_{C}$ & 0.19 & -2.45 & -38.67 & 4.33 & -1.77 \\
& 0.38 & -2.09 & -25.13 & 6.21 & -3.01 \\
& 0.89 & 17.90 & -33.31 & 13.11 & -5.41 \\
$h_{T}$ & 0.19 & -2.96 & 24.53 & 7.71 & -6.12 \\
& 0.38 & -2.25 & -9.91 & 16.67 & -12.05 \\
& 0.89 & 0.40 & -0.39 & 6.62 & -29.06 \\
\hline
\end{tabular}



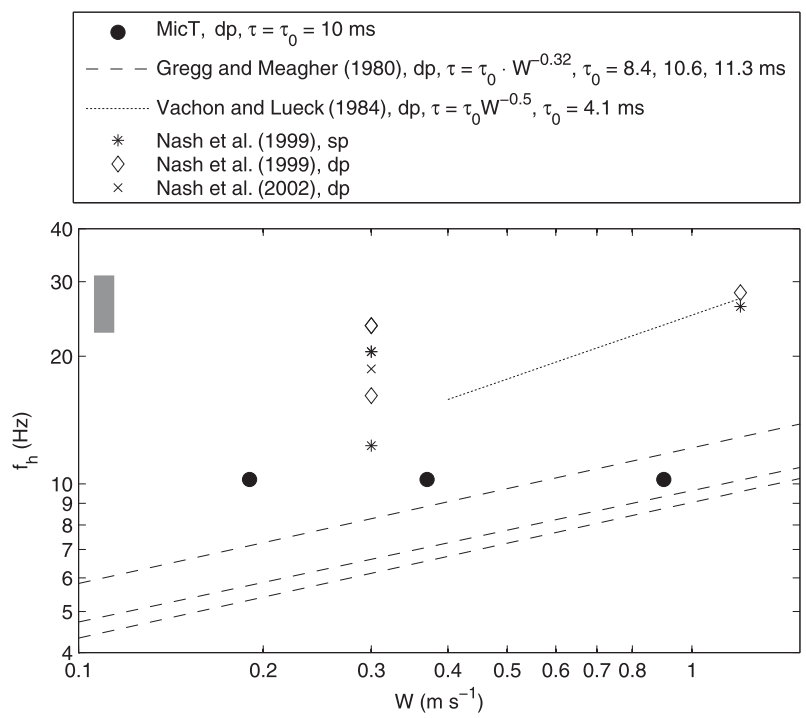

FIG. 6. Half-power frequencies $f_{h}$ of reported FP07 frequency responses and their profiling speed dependence. The legend indicates the defining parameters; $\mathrm{dp}$ and $\mathrm{sp}$ are short for double-pole and single-pole responses, respectively. The length of the lines indicates the investigated speed range. The gray bar on the left corresponds to the specifications given by the manufacturer (details in text).

\section{a. MicT sensor frequency response}

The frequency response characteristics of the FP07 are not known exactly. An overview of the reported frequency responses, including the manufacturer's specification, is given in Fig. 6, in which we compare half-power frequencies $f_{h \text {,MicT }}$ and their profiling speed dependence. The findings differ in time constants, velocity scaling of the time constants, and the shape of the response (double or single pole). We use here a doublepole response for which we deduced $\tau_{\mathrm{dp}}=10 \mathrm{~ms}$ (without velocity dependence) and that has a half-power frequency of $10.2 \mathrm{~Hz}$. Our half-power frequency is lower than the manufacturer's specification but well within the range of findings of other researchers.

Different half-power frequencies between sensors might be partly explained by different geometries of the glass coating surrounding the sensors, which are not perfectly identical for every individual sensor (Gregg and Meagher 1980). However, varying time constants may also be a result of differences in the experimental setup. Therefore, we discuss two different approaches for determining the frequency response of the MicT, in particular the methods of the manufacturer GE and Nash et al. (1999) and Nash and Moum (2002).

GE states a "nominal" response time of $7 \mathrm{~ms}$, determined by dropping the sensor from air into water (at $W<0.1 \mathrm{~m} \mathrm{~s}^{-1}$ ) and measuring the time until the signal reaches $63 \%$ of the difference between the initial and final values (J. Lukie, GE Energy, 2012, personal communication). They do not specify the shape of the response (i.e., whether it is a single- or double-pole response). The corresponding time constants for a singleand a double-pole frequency response are $\tau_{\mathrm{sp}}=7 \mathrm{~ms}$ (corresponding to their nominal response time) and $\tau_{\mathrm{dp}}=3.3 \mathrm{~ms}$, respectively, with half-power frequency points at 22.7 and $31 \mathrm{~Hz}$. Dropping a dry sensor from air into water always underestimates the time constant because the water boundary layer (through which heat must be diffused) has not had time to develop before the sensors first touch the water (Lueck et al. 1977). Thus, it is not surprising that our own measurements in water result in a larger time constant.

Nash et al. (1999) and Nash and Moum (2002) use the $T$ dependence of the $C$ signal to measure the in situ $T$ gradient spectrum with a four-point conductivity microstructure probe (Head 1983). The frequency re-

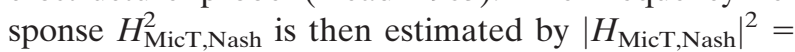
$\psi_{\mathrm{Tz}} / \psi_{\mathrm{Cz}}$, where $\psi_{\mathrm{Tz}}$ is the $T$ gradient spectrum measured with the MicT and $\psi_{\mathrm{Cz}}$ is the $T$ gradient spectrum measured with the microstructure conductivity probe. This procedure assumes that the conductivity sensor is infinitely fast. If this was not the case, the MicT half-power frequency response would be overestimated and the MicT would have to be corrected for the MicC response as $\left|H_{\text {MicT,Nash,corr }}\right|^{2}=\left|H_{\text {MicT,Nash }}\right|^{2}\left|H_{\text {MicC }}\right|^{2}$. For example, if we assume $\left|H_{\text {MicT,Nash }}\right|^{2}$ to be a double-pole frequency response with a half-power frequency $f_{h, \text { MicT,Nash of }}$ 18.7 Hz as in Nash and Moum (2002) and $f_{h, \mathrm{MicC}}=47 \mathrm{~Hz}$ for $H_{\mathrm{MicC}}$ as determined for our MicC, $H_{\mathrm{MicT} \text {,Nash,corr }}$ would have a smaller half-power frequency of $f_{h}=17 \mathrm{~Hz}$ but still larger than our MicT estimate of $f_{h}=10.2 \mathrm{~Hz}$. A possibly neglected MicC frequency response thus does not explain the difference between our MicT halfpower frequency and the one of Nash and Moum (2002). We expect this difference could rather be attributed to the large scatter in $f_{h}$ between different FP07 sensors that results from manufacturing.

\section{b. MicC sensor frequency response}

The observed frequency response with a time constant of $2.2 \mathrm{~ms}$ might be induced by the signal processing or by the interaction of the moving sensor with the fluid environment. Possible contributions from the signal processing are the antialiasing (AA) filter and the preemphasis and its deconvolution (Mudge and Lueck 1994). The boundary layer over the sensor electrodes may be another possible cause for the observed frequency response. In the following we show that contributions from the signal processing are not large enough to explain the observed frequency response and that the delayed flushing of the viscous boundary layer 

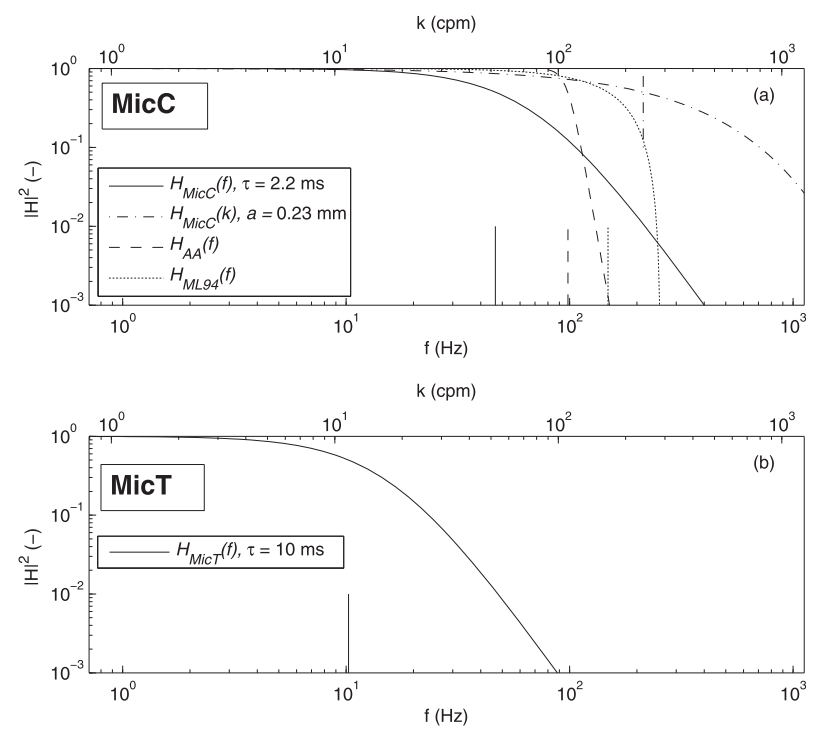

FIG. 7. (a) Response functions for the MicC sensor. The relation between wavenumber (top axis) and frequency (bottom axis) corresponds to a profiling speed of $0.89 \mathrm{~m} \mathrm{~s}^{-1}$. Measured interface thicknesses were corrected for the frequency response $H_{\mathrm{MicC}}(f)$ and the wavenumber response $H_{\mathrm{MicC}}(k)$ as described in section $4 \mathrm{~b}$. The expected frequency responses of the antialiasing filter $H_{\mathrm{AA}}(f)$ and the preemphasis and deconvolution method $H_{\mathrm{ML} 94}(f)$ are also shown. Corresponding half-power frequencies are indicated as vertical lines. (b) Frequency response of the MicT sensor $H_{\mathrm{MicT}}(f)$. Note that $H_{\mathrm{AA}}(f)$ and $H_{\mathrm{ML} 94}(f)$ apply also for MicT but are not shown for clarity.

reasonably predicts the time scale of the observed frequency response.

\section{1) AA FILTER}

At very high frequency, the response is speed dependent because AA filters must be employed before the analog data are sampled. Spatial features with very small spatial scales will appear at high frequency and will be attenuated (and lagged) by the AA filters if the product $W k$ exceeds the half-power frequency of the AA filters. The AA filter is an eight-pole low-pass Butterworth filter with a sharp cutoff at the half-power frequency $f_{h, \mathrm{AA}}=98 \mathrm{~Hz}$. Its transfer function is

$$
H_{\mathrm{AA}}(f)=\prod_{i=1}^{4}\left[1+j \alpha_{i}\left(\frac{f}{f_{h, \mathrm{AA}}}\right)-\left(\frac{f}{f_{h, \mathrm{AA}}}\right)^{2}\right],
$$

where $\alpha_{i}=1.9616,1.6629,1.1112$, and 0.3902 for $i=(1$, 2, 3, 4) (RSI; Fig. 7a, dashed line). Our double-pole frequency response $H_{\mathrm{MicC}}(f)$ has a half-power frequency $f_{h, \mathrm{MicC}}=47 \mathrm{~Hz}$ (Fig. 7a), which corresponds to approximately $0.5 f_{h, \mathrm{AA}}$. Thus, the AA filter does not explain the observed frequency response.

\section{2) SignAl PREEMPHASIS AND ITS DECONVOLUTION}

The microstructure signals are preemphasized and deconvolved (Mudge and Lueck 1994). Therefore, the signal and its preemphasized derivative are summed in the analog domain before sampling. The preemphasis is equivalent to an "inverse" filter enhancing the highfrequency portion of the signal. The subsequent deconvolution is achieved with a single-pole Butterworth low-pass filter in the discrete domain. The coefficients of this filter are derived using the bilinear transformation (Proakis and Manolakis 1988). The low-frequency characteristics of the discrete domain filter match very closely to those of the equivalent continuous domain filter, but their characteristics depart for frequencies larger than about one-third of the Nyquist frequency. Thus, the deconvolution filter is not the exact inverse of the preemphasis filter. The ratio of the deconvolution filter to that of the preemphasis filter can be considered as a frequency response, which is shown in Fig. 7a denoted by ML94 (dotted line). Its half-power frequency $f_{h, \text { ML } 94}$ is $148 \mathrm{~Hz}$, much larger than $f_{h, \mathrm{AA}}$ and $f_{h, \mathrm{MicC}}$ and thus this part of the signal processing cannot explain our deduced frequency response for the $C$ sensor.

\section{3) BOUNDARY LAYER}

In the viscous boundary layer surrounding the sensor electrodes, the fluid velocity adjusts from its free-stream value down to zero at the sensor's surface, satisfying the no-slip boundary condition. Thus, in the viscous boundary layer, the renewal of water by the ambient flow is delayed, causing retardation of the measured signal relative to the free stream. We use a simple model (appendix B) where the time scale of the response results from the advection of fluid into the viscous boundary layer. Assuming a single-pole response function, the model predicts a time constant $\tau_{\mathrm{sp}}=2 \mathrm{~ms}$. Our measurements suggest $\tau_{\mathrm{dp}}=2.2 \mathrm{~ms}$ for a double-pole response, which corresponds to single-pole time constant of approximately $6 \mathrm{~ms}$. Considering the simple assumptions made for our model, the agreement is reasonable. In addition, we estimate that $44 \%$ of the measured signal is from the viscous boundary layer and thus processes within the viscous boundary layer as described above are important. Hill and Woods (1988) have previously suggested that the conductivity sensor has only a wavenumber response. They used faster profiling speeds of $0.54-1.89 \mathrm{~m} \mathrm{~s}^{-1}$ with a larger electrode spacing of $a \sim$ $2 \mathrm{~mm}$. For their configuration, the viscous boundary layer contribution to the measured signal is much smaller than it is for our MicC and ranges between $10 \%$ and $17 \%$, depending on $W$. This may explain why we observe 
a frequency response while Hill and Woods (1988) did not.

The frequency response of the MicC becomes important when the size, and spacing, of the electrodes is reduced in an attempt to reach extremely small spatial resolution. The frequency response resulting from the viscous boundary layer negates the benefit of finer spatial resolution. The spatial resolution of the MicC increases with slower profiling, if only the frequency response is considered. At some transition profiling speed $W^{*}$, the wavenumber response, which is independent of $W$, exceeds the spatial resolution. We estimate $W^{*}$ by $f_{h, \mathrm{MicC}} / k_{h, \mathrm{MicC}}$, where the half-power wavenumber $k_{h, \text { MicC }}$ is given by $\left|H_{\text {MicC }}\left(k_{h, \text { MicC }}\right)\right|^{2}=0.5$. For $\tau_{\mathrm{dp}}=2.2 \mathrm{~ms}$ and $a=0.23 \mathrm{~mm}$ we have $f_{h, \mathrm{MicC}}=$ $47 \mathrm{~Hz}, k_{h, \mathrm{MicC}}=240 \mathrm{cpm}$, and consequently $W^{*}=$ $0.19 \mathrm{~m} \mathrm{~s}^{-1}$, which is our slow profiling speed. For faster profiling speeds, the frequency response will dominate the spatial smoothing and consequently the spacing between the electrodes should be increased in order to reduce the effect of the frequency response.

\section{Implications for double-diffusive fluxes}

\section{a. Molecular fluxes through interfaces}

Heat and salt fluxes through double-diffusive staircases are often approximated by the molecular fluxes through interfaces (e.g., Timmermans et al. 2008). For their quantification it is thus important to determine the gradients within the interface cores. According to the definition of $h_{T}$ and $h_{S}$ these gradients can be expressed as $\Delta T / h_{T}$ and $\Delta S / h_{S}$. Because $T$ has an influence on the measured $C$ signal, this needs to be corrected for when estimating the finescale salinity field. For the computation of $S$ from $T$ to $C$ and the conversion from $h_{C}$ to $h_{S}$ we refer to Sommer et al. (2013, manuscript submitted to $J$. Geophys. Res.). In Lake Kivu the conductivity gradient $\partial C / \partial z$ is mainly determined by $\partial S / \partial z$, with a contribution of only about $10 \%$ by $\partial T / \partial z$. Consequently, $h_{C}$ and $h_{S}$ are similar with $h_{C}$ being only $3.5 \%$ larger than $h_{S}$ on average. Mean interface thicknesses (with 10th and 90th percentiles in parentheses) are $\overline{h_{S}}=6.8 \mathrm{~cm}(0.6,14.4)$ and $\overline{h_{T}}=9.9 \mathrm{~cm}(3.1,17.8)$. This indicates that a gravitationally unstable boundary layer is present on either side of the interface as predicted by Linden and Shirtcliffe (1978), Worster (2004), and Carpenter et al. (2012a).

By definition of $h_{T}$ and $h_{S}$, molecular heat and salt fluxes through interfaces are then

$$
\begin{aligned}
& F_{H}=c_{p} \rho \kappa_{T} \Delta T h_{T}^{-1} \quad\left(\mathrm{~W} \mathrm{~m}^{-2}\right) \quad \text { and } \\
& F_{S}=\rho \kappa_{S} \Delta S h_{S}^{-1} \quad\left(\mathrm{~g} \mathrm{~m}^{-2} \mathrm{~s}^{-1}\right),
\end{aligned}
$$

where $c_{p}\left(\mathrm{~J} \mathrm{~kg}^{-1} \mathrm{~K}^{-1}\right)$ is the specific heat capacity of water, $\rho\left(\mathrm{kg} \mathrm{m}^{-3}\right)$ is its density, and $\kappa_{T}\left(\mathrm{~m}^{2} \mathrm{~s}^{-1}\right)$ and $\kappa_{S}\left(\mathrm{~m}^{2} \mathrm{~s}^{-1}\right)$ are the molecular diffusivity of heat and salt, respectively. Molecular fluxes through interfaces are inversely proportional to the interface thickness. Consequently, small interface thicknesses cause large fluxes, and the average values are sensitive to the occurrence of those individual thin interfaces. Because the sensor response correction reduces interface thicknesses disproportionately for small interface thicknesses (Fig. 5), the correction is important for calculating average fluxes. Small absolute errors in the thin interface thicknesses can cause large absolute errors in the fluxes. Therefore, we excluded the 1 st percentile of $h_{T}\left(h_{T}<1.5 \mathrm{~cm}\right)$ and $h_{S}\left(h_{S}<0.16 \mathrm{~cm}\right)$ from flux calculations but, for completeness, we also provide the values without this restriction.

The molecular heat flux through the double-diffusive interfaces, averaged over all measured interfaces in Lake Kivu staircases, is $78 \mathrm{~mW} \mathrm{~m}^{-2}(11173)$ and $81 \mathrm{~mW} \mathrm{~m}^{-2}$ including the 1st percentile of $h_{T}$. The average heat flux increase resulting from interface thickness correction for slow, medium, and fast profiling is $5.8 \%, 6.9 \%$, and $22.3 \%$, respectively. For comparison, the correction of Vachon and Lueck (1984) yields increases of $4.8 \%$, $6.1 \%$, and $8.1 \%$, respectively, and thus underestimates the heat flux in particular for fast profiling. The average salt flux is $0.81 \mu \mathrm{g} \mathrm{m}^{-2} \mathrm{~s}^{-1}(0.09,1.59)$ and rises to $1.04 \mu \mathrm{g} \mathrm{m}^{-2} \mathrm{~s}^{-1}$ if we include the 1 st percentile. The interface thickness correction increases the average salt flux by $9.6 \%, 12.1 \%$, and $28.0 \%$ for slow, medium, and fast profiling, respectively. If only the wavenumber response of Hill and Woods (1988) is corrected for, increases of $6.9 \%, 4.4 \%$, and $1.0 \%$, respectively, are obtained. This is in agreement with our expectations and Figs. $4 \mathrm{~b}$ and $4 \mathrm{~d}$. The smallest measured interface thicknesses are detected for slow profiling because measured interfaces are influenced by the frequency response. The smaller the interface thickness, the larger is the correction by the wavenumber response, which increases the average flux foremost for slow profiling.

In summary, our findings show that average fluxes through interfaces are sensitive to both the response characteristics of the sensors and the large contribution by a few very thin interfaces.

\section{b. Interface thickness ratio}

The interface thickness ratio $r=h_{T} / h_{S}$ is another important physical parameter for double diffusion. It can be used for testing theories of double-diffusive boundary layers (Carpenter et al. 2012b) and it appears in the density flux ratio $R_{F}$ of molecular fluxes through interfaces, defined as 


$$
R_{F}=\frac{\beta \kappa_{S} \Delta S h_{S}^{-1}}{\alpha \kappa_{T} \Delta T h_{T}^{-1}}=\tau r R_{\rho},
$$

where $\beta\left(\mathrm{kg} \mathrm{g}^{-1}\right)$ is the haline coefficient of contraction, $\alpha\left(\mathrm{K}^{-1}\right)$ is the thermal coefficient of expansion, $\tau=$ $\kappa_{S} / \kappa_{T} \sim 0.01$ is the diffusivity ratio, and $R_{\rho}=\beta \Delta S / \alpha \Delta T$ is the density ratio.

Because the correction for both $T$ and $C$ interfaces reduces the interface thickness in a nonlinear way, the effect of the correction on the ratio $r$ is less clear. The average $r$ is $\bar{r}=2.6(0.9,5.5)$ and rises to 2.9 by including the 1st percentile. Note that $\bar{r}$ is much larger than $\overline{h_{T}} / \overline{h_{S}}=1.5$ because $r$ is very sensitive to the occurrence of small $h_{S}$ (Fig. 4f). Here, $\bar{r}$ increases by $4.3 \%, 6.6 \%$, and $5.2 \%$ for slow, medium, and fast profiling, respectively.

\section{Conclusions and outlook}

The distributions of interface thicknesses of the doublediffusive staircases in Lake Kivu have been used to estimate the frequency responses of conductivity and temperature microstructure sensors. Our method does not rely on knowledge of the true $T$ and $C$ structure but takes advantage of the speed dependency of the frequency response on the spatial resolution. Doublediffusive interfaces provide distinct step changes in $T$ and $C$ for measuring the response of very fast sensors. In particular for $C$, the thickness of the thinnest interfaces was smaller than $1 \mathrm{~mm}$ and thus close to the estimated Batchelor scales. Such sharp transitions are difficult to maintain in laboratory experiments. The response time constant of the SBE-7 conductivity sensor was found to be $2.2 \mathrm{~ms}$ assuming a double-pole frequency response. This is, to our knowledge, the first observation of a frequency response for conductivity microstructure sensors that is thought to result from the interaction of the moving sensor with its fluid environment. The time constant of the frequency response is of the same order as the advection time scale of salt into the viscous boundary layer on the sensor. For the FP07, the time constant of the double-pole frequency response was found to be $10 \mathrm{~ms}$ (omitting any velocity scaling), which is in the range of previously observed values.

Measured $T$ and $C$ interface thicknesses were corrected for the sensor responses and the effect of these corrections on the heat flux $F_{H}$, the salt flux $F_{S}$, and the ratio of the temperature to salt interface thickness $r$ was studied. We showed that all three parameters are sensitive to the sensor response correction.

Determining the frequency response of a sensor by measuring physical structures at different speeds is not restricted to double-diffusive interfaces. Turbulence in stratified water bodies might also provide the small-scale structures needed to infer sensor responses. In particular, the integrals over gradient spectra of $T$ and $C, \chi_{T}$ and $\chi_{C}$, respectively, might be an appropriate choice for testing sensor responses. The distributions of $\chi_{T}$ and $\chi_{C}$ should be profiling speed independent, if the sensor response corrections were applied correctly. If speed dependency is observed, a frequency response might have been neglected or over- or underestimated. It is well possible that this requires a larger dataset than the one used in Lake Kivu to assume representative and constant distributions of $\chi_{T}$ and $\chi_{C}$ for a particular study area, the time period of the study, and for each profiling speed. However, when the instrument design and the measurement time frame allows, it might be worth designing field experiments such that more than one profiling speed is used. This will increase the confidence in microstructure frequency responses and finally also in turbulent flux estimates.

Acknowledgments. The authors thank Michael Schurter for his effort during field work; Natacha and Tom Tofield for their hospitality and organizational support in Rwanda; Fidèle Kanamugire, owner of the transportation vessel Gloria, and his crew for their help on the lake; and Hanspeter Schmid for his valuable advice on signal processing. The manuscript has greatly benefitted from the comments of the two anonymous referees. The project was supported by the Swiss National Science Foundation under Grant 200021-122183 (Lake Kivu-turbulence and double diffusion in permanent stratification).

\section{APPENDIX A}

\section{Interface Detection Algorithm}

We explain the four conditions of the interface detection algorithm using three consecutive $T$ interfaces shown in Fig. A1, but identical arguments hold for $C$. In Fig. A1, the middle interface $I_{m}$ is sandwiched between two mixed layers $\mathrm{ML}_{a}$ (above) and $\mathrm{ML}_{b}$ (below), respectively, followed by two more interfaces $I_{a}$ (above) and $I_{b}$ (below).

\section{a. Condition (i)}

Whenever

$$
\frac{\partial \bar{T} / \partial z}{\partial \bar{T} / \partial z}>1.8
$$

(with both $\partial \bar{T} / \partial z>0$ and $\partial \overline{\bar{T}} / \partial z>0$ ), the data are considered a candidate interface and delineated by circles. 


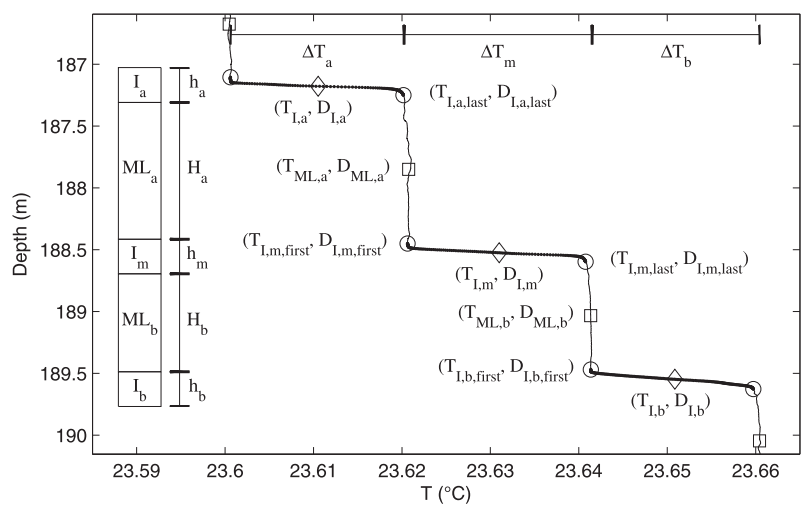

FIG. A1. Example of a temperature microstructure profile section and relevant parameters for the interface detection algorithm. For clarity, the interface thicknesses $h_{a}, h_{m}$, and $h_{b}$ on the left are indicated larger than calculated.

Here $\bar{T}$ is the slightly smoothed $T$ obtained by applying a first-order Butterworth filter with a cutoff frequency of $f_{c}=W / \theta$, where $\theta$ is a length scale much smaller than the average mixed-layer thickness $H$; $\bar{T}$ is the background profile, determined by a moving average of over a vertical distance $\Theta \gg H$. For Lake Kivu, $H \approx 0.8 \mathrm{~m}$ and we chose $\theta=0.04$ and $\Theta=6 \mathrm{~m}$. The interfacial points (Fig. A1) appear almost as a single thick line because their spacing is tight. Below we define the staircase parameters to be determined by the algorithm for $I_{m}$ and $\mathrm{ML}_{b}$. Identical notation is valid for $I_{a}, I_{b}$, and $\mathrm{ML}_{a}$ with adjusted indices.

\section{$\left(T_{I, m, \text { first }}\right.$, \\ $\left.D_{I, m \text {,first }}\right): \quad$ temperature and depth of the first point of the series of interface points $I_{m}$ \\ $\left(T_{I, m, \text { last }}\right.$, \\ $\left.D_{I, m, \text { last }}\right): \quad$ temperature and depth of the last point \\ $\left(T_{\mathrm{ML}, b}\right.$, of the series of interface points $I_{m}$ \\ $\left.D_{\mathrm{ML}, b}\right)$ \\ $H_{b}^{*}$ : \\ $H_{b}$ : \\ $\Delta T_{m}$ : \\ $\left(T_{I, m}, D_{I, m}\right)$ : \\ temperature and depth of the center of $\mathrm{ML}_{b}$ preliminary thickness of $\mathrm{ML}_{b}$ \\ final thickness of $\mathrm{ML}_{b}$ \\ temperature step across $I_{m}$ \\ temperature and depth of the center of $I_{m}$ \\ $h_{m}$ : \\ thickness of interface $I_{m}$}

The individual staircase parameters are then calculated as follows, starting from the points determined by condition (i):

- $D_{\mathrm{ML}, b}=\left(D_{I, m, \text { last }}+D_{I, b, \text { first }}\right) / 2$.

- $H_{b}^{*}=D_{I, b, \text { first }}-D_{I, m, \text { last }}$.

- $T_{\mathrm{ML} b}$ is calculated by averaging the temperature within the interval $D_{\mathrm{ML}, b} \pm H_{b}^{*} / 2$.

- Steps one to three are carried out identically for $\mathrm{ML}_{a}$, defining $D_{\mathrm{ML}, a}, H_{a}^{*}$, and $T_{\mathrm{ML}, a}$.
- $\Delta T_{m}=T_{\mathrm{ML}, b}-T_{\mathrm{ML}, a}$.

- $T_{I, m}=\left(T_{\mathrm{ML}, a}+T_{\mathrm{ML}, b}\right) / 2$.

- $D_{I, m}$ is determined by averaging the depths of the points within the interval $T_{I, m} \pm \Delta T_{m} / 4$. Because measurement points are approximately equally spaced for a free-falling profiler with constant sampling frequency, averaging is unproblematic.

- $(\partial T / \partial z)_{\text {fit }, I, m}$ : A straight line is fitted to the data within the interval $T_{I, m} \pm \Delta T_{m} / 4$. At least two fit points are necessary. The slope of this line determines $(\partial T / \partial z)_{\text {fit } I, m}$.

- $h_{m}=\Delta T_{m} /(\partial T / \partial z)_{\text {fit }, I, m}$.

- The interface thicknesses $h_{a}$ and $h_{b}$ are calculated identically to the previous step.

- The final mixed-layer thicknesses $H_{b}$ and $H_{a}$ (replacing the preliminary mixed-layer thicknesses $H_{b}^{*}$ and $\left.H_{a}^{*}\right)$ are given as $H_{b}=\left(D_{I, b}-D_{I, m}\right)-\left(h_{b}+h_{m}\right) / 2$ and $H_{a}=\left(D_{I, m}-D_{I, a}\right)-\left(h_{m}+h_{a}\right) / 2$. This definition ensures that the thickness of all layers and interfaces sum to the total vertical length of the staircase.

\section{b. Condition (ii)}

Both mixed-layer gradients adjacent to the interface are small compared to the background gradient. For the mixed-layer $\mathrm{ML}_{b}$, this can be written as

$$
-0.4<\frac{(\partial T / \partial z)_{\mathrm{fit}, \mathrm{ML}, b}}{(\partial \overline{\bar{T}} / \partial z)_{\mathrm{ML}, b}}<0.4 .
$$

Here, $(\partial T / \partial z)_{\mathrm{fit}, \mathrm{ML}, b}$ is the slope of a linear fit through the mixed-layer points within the interval $D_{\mathrm{ML}, b} \pm H_{b} / 2$; $(\partial \bar{T} / \partial z)_{\mathrm{ML}, b}$ is the background gradient averaged over the same interval.

\section{c. Condition (iii)}

Only if all four sensors detect the same interface according to arguments (i) and (ii), the step parameters, as defined in (i), are saved. The condition for detecting the same interface is that all four interface centers are located within a vertical interval of $10 \mathrm{~cm}$, which is the minimum distance between interface centers.

\section{d. Condition (iv)}

The definition of an interface thickness only makes sense if the interfaces are approximately linear in their cores. Therefore, we introduced the rejection parameter $M=T_{\mathrm{rms}} / \Delta T$ where $T_{\mathrm{rms}}$ is the root-mean-square deviation of the measured $T$ from the linear fit through the central $50 \%$ of the interface. Based on visual inspection we chose to discard steps with $M>0.03$. Figure A2 shows two typical examples of rejected $C$ interfaces, whereas both $T$ interfaces are accepted. In Fig. A2a, the inversion in the $C$ interface is probably caused by a 

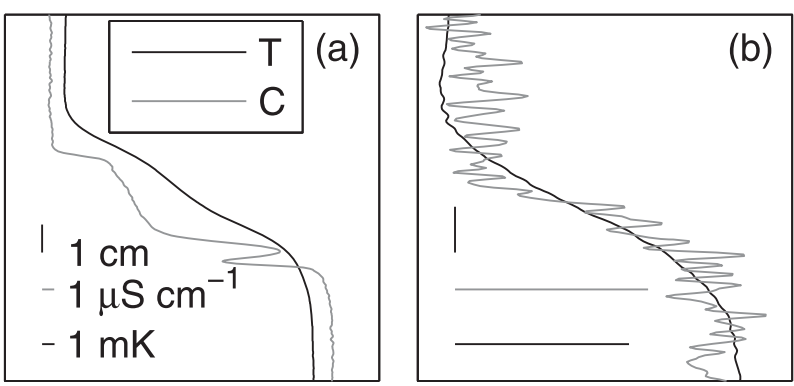

FIG. A2. Examples of rejected $C$ interfaces and accepted $T$ interfaces from in Lake Kivu. The scales for depth, $T$, and $C$ are indicated. (a) Inversion in $C$ interface. (b) $C$ step dominated by instrument noise.

physical disturbance of the interface, which has already diffused out in the $T$ interface. In Fig. A2b, the inversions are caused by noise from the MicC sensor. As a result of these rejection criteria, the total number of steps decreased by $14 \%$ and $54 \%$ for $T$ and $C$, respectively. Conductivity was more affected by the rejection criteria because inversions in $C$ persist longer because of the smaller diffusivity of salt compared to $T$ (Fig. A2a) and because the ratio of noise to step size is on average about 10 times larger for $C$ than for $T$ (Fig. A2b).

\section{APPENDIX B}

\section{A Simple Model of the MicC Frequency Response}

The MicC consists of two electrodes with a constantamplitude sinusoidal voltage difference applied between them. The resulting electric field drives a current through the fluid that is inversely proportional to its resistance. The spatial distribution of the field lines determines the measurement volume, which consists of a free-stream portion, a viscous boundary layer portion, and a diffusive boundary layer portion, where the latter is negligible. Conductivity changes in the free stream are sensed immediately, whereas in the viscous boundary layer the flow is delayed. This delay causes the sensor to have a frequency response. However, the frequency response is only observed if the contribution of the viscous boundary layer to the total resistance is significant. Our model consists of two parts: (i) we estimate the time scale of how fast changes in $C$ are advected into the viscous boundary layer and (ii) we estimate the contribution of the boundary layer to the total resistance.

Figure B1a shows our model setting for part (i). We only consider one of the two MicC electrodes, which is represented by a solid plane located at $(x, y, z=0)$. The electrode starts at $x=0$ and ends at $x=d$ with the flow in the $x$ direction. We only consider horizontal advection into the viscous boundary layer and neglect molecular diffusion because it can be shown to be small. At $t=0$, the front of a rapid change of strength $C_{0}$ is taken to be located at $x=0$ and is then advected across the sensor. We neglect the change in boundary layer thickness $\delta_{\nu}$ over the length of the sensor and just look at the changes in the concentration profile at $x=d / 2$ (Fig. B1b), which is assumed to represent the mean along $x$. Furthermore, we assume a linear velocity profile $u(z)$ in the boundary layer and constant velocity $W$ in the free stream (Fig. B1a), so that

$$
u(z)=\left\{\begin{array}{ll}
W, & z>\delta \\
W z / \delta, & 0<z<\delta
\end{array} .\right.
$$

At any time, the horizontal location $X$ of the front at height $z$ within the viscous boundary layer (Fig. B1b) is given by

$$
X=\frac{W z}{\delta} t
$$

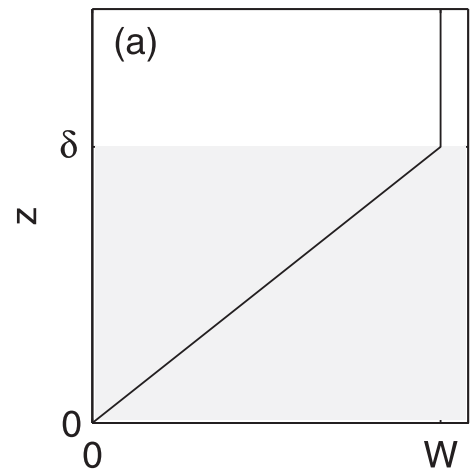

$\mathrm{u}$
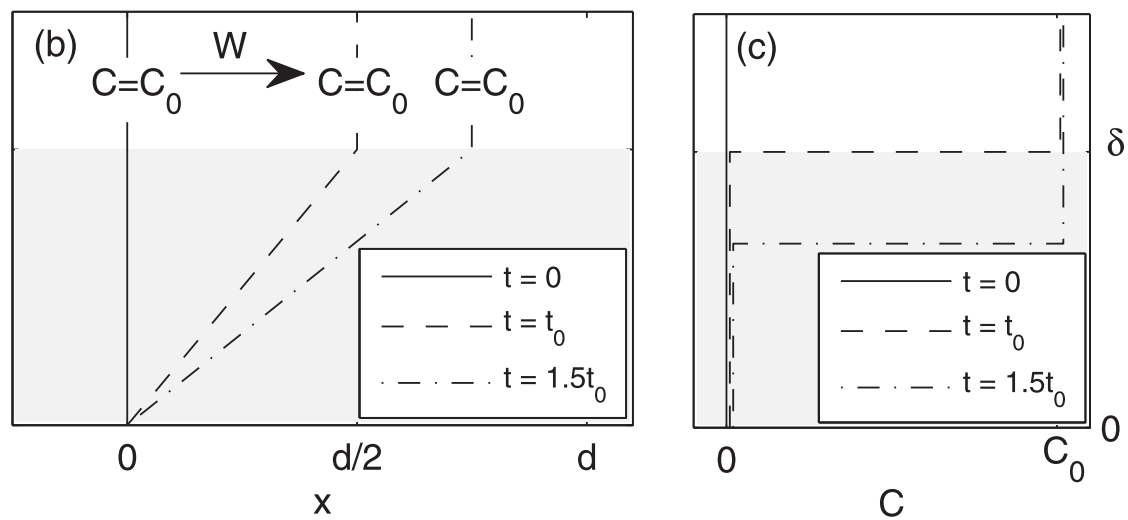

FIG. B1. (a) Velocity profile in the viscous boundary layer (gray area) as a function of distance $z$ from the sensor surface. (b) Location of the conductivity front in the viscous boundary layer at three different times with $t_{0}$ being the time after which the front in the free stream has reached the center $d / 2$ of the sensor. (c) Conductivity profiles at $x=d / 2$ at the same times as in (b). 
The sensor will see a concentration profile with front of concentration $C_{0}$ moving downward as the front is advected horizontally past the point $x=d / 2$, which happens after the time $t=t_{0}=d /(2 W)$ (Fig. B1c). The height of the front above the sensor $Z$ at $x=d / 2$ is then given by

$$
Z=\frac{d \delta}{2 W t}
$$

with $t>t_{0}$. Note that the speed $d Z / d t$ of the front is decreasing with $t^{-2}$. The mean $C$ in the viscous boundary layer as a function of time is then

$$
C_{m}(t)=\frac{1}{\delta} \int_{0}^{\delta} C(z, t) d z=\frac{1}{\delta} C_{0}(\delta-Z)=C_{0}\left(1-\frac{d}{2 W t}\right)
$$

for $t>t_{0}$. Note that $C_{m}(t)$ is independent of $\delta$. This relation allows estimating the time scale of the frequency response for concentration changes within the viscous boundary layer. Setting $C_{m}\left(\tau_{\mathrm{sp}}+t_{0}\right) / C_{0}=1-e^{-1}$ provides a single-pole time constant $\tau_{\mathrm{sp}}$ of

$$
\tau_{\mathrm{sp}}=\frac{d}{2 W}(e-1) .
$$

For $d=0.9 \mathrm{~mm}$ (diameter of the MicC electrode) and $W=0.38 \mathrm{~m} \mathrm{~s}^{-1}$, we get $\tau_{\mathrm{sp}}=2 \mathrm{~ms}$. Our double-pole frequency response with $\tau_{\mathrm{dp}}=2.2 \mathrm{~ms}$ fulfills the same condition at $\tau_{\mathrm{sp}} \sim 6 \mathrm{~ms}$. This simple model thus appears to provide the approximate time scale of the MicC frequency response, but it also predicts a velocity scaling $\tau_{\mathrm{dp}} \sim W^{-1}$, which is not supported by our data (section $4 \mathrm{~b}$ ). In the following we show that the viscous boundary layer represents a significant fraction of the measured signal.

For part (ii), the MicC sensor measures the electric current $J$ (A) while applying a constant-amplitude sinusoidal voltage difference $\Delta V(\mathrm{~V})$ between the electrodes. The current is given by $J(t)=\Delta V / R(t)$, where $R(t)(\Omega)$ is the resistance between the electrodes, which changes over time depending on the conductivity $C$ in the measurement volume. We divide the measurement volume and the resistance into a free-stream portion and a viscous boundary layer portion:

$$
R=R_{\mathrm{FS}}+R_{\delta}
$$

We assume that the resistance of the free stream is instantaneously reacting to changes of $C$ in the flow, whereas the resistance in the viscous boundary layer is only changing after the $C$ variations of the free stream have been advected into the viscous boundary layer by the process described in part (i). The time constant determined in part (i) is only observed if the contribution
$P_{\delta}=R_{\delta} / R$ to the total resistance $R$ is significant. The resistance of the viscous boundary layer is proportional to the electric potential difference $\Delta V_{\delta}$ across this layer and therefore

$$
P_{\delta}=\frac{R_{\delta}}{R}=\frac{\Delta V_{\delta}}{\Delta V} .
$$

The electric potential $V(\mathrm{~V})$ is given by the geometry of the electrodes. Here, we represent the electrodes by two metal spheres of diameter $d$ with their centers at $y=$ $-a-d / 2$ and $y=a+d / 2$. The electrodes carry the charges $q(\mathrm{C})$ and $-q$ and are surrounded by a viscous boundary layer. The viscous boundary layer thickness is

$$
\frac{\delta}{d} \approx \frac{2}{\sqrt{\mathrm{Re}}}
$$

where $\operatorname{Re}=W d / \nu$ is the Reynolds number with profiling speed $W$ and the kinematic viscosity of water $\nu$ (Schlichting and Gersten 2003, p. 173, Fig. 7.3).

The charges cause an electric potential outside the spheres that we assume is equal to the potential of two hypothetical point charges $q$ and $-q$ located at the centers of the spheres. We simplify the problem further by only considering $V$ on the line connecting the two electrodes. The potential $V$ evaluated on this line at $-a-d / 2<y<a+d / 2$ is

$$
V(y)=\frac{q}{4 \pi \varepsilon}\left(\frac{1}{a+d / 2-y}-\frac{1}{a+d / 2+y}\right),
$$

where $\varepsilon\left(\mathrm{A} \mathrm{s} \mathrm{m}^{-1} \mathrm{~V}^{-1}\right)$ is the permittivity, which is assumed to be constant. The potential is antisymmetric relative to $y=0$ and therefore we restrict the problem to $y>0$. The potential differences across the free-stream layer and the viscous boundary layer are then

$$
\begin{gathered}
\Delta V_{\mathrm{FS}}=V(0)-V(a-\delta) \quad \text { and } \\
\Delta V_{\delta}=V(a-\delta)-V(a)
\end{gathered}
$$

where $\Delta V=2\left(\Delta V_{\mathrm{FS}}+\Delta V_{\delta}\right)$ and $V(0)=0$ by symmetry. Substituting Eq. (B2) into Eq. (B1) gives

$$
P_{\delta}=\frac{\Delta V_{\delta}}{\Delta V_{\mathrm{FS}}+\Delta V_{\delta}}=1-\frac{(2 a+d / 2-\delta)^{-1}-(d / 2+\delta)^{-1}}{(2 a+d / 2)^{-1}-(d / 2)^{-1}}
$$

For $a \rightarrow \delta, P_{\delta} \rightarrow 1$, as the viscous boundary layer occupies the entire spacing between the electrodes. For $a \gg \delta \gg d / 2, P_{\delta} \rightarrow 1-d /(2 \delta)$. This means that, even when the electrode spacing is large, the resistance in the viscous boundary layer contribution is still significant if 
the electrodes are small compared to the viscous boundary layer thickness.

For $d=0.86 \mathrm{~mm}, a=0.23 \mathrm{~mm}, W=0.38 \mathrm{~m} \mathrm{~s}^{-1}$, and $\nu=9 \times 10^{-7} \mathrm{~m}^{2} \mathrm{~s}^{-1}$ we get $\mathrm{Re}=363, \delta=90 \mu \mathrm{m}$, and $P_{\delta}=0.44$. Thus, the resistances of the fluid in the viscous boundary layer and in the free stream are almost equal, and consequently, processes occurring in the viscous boundary layer such as that described in part (i) are important.

\section{REFERENCES}

Carpenter, J. R., T. Sommer, and A. Wüest, 2012a: Stability of a double-diffusive interface in the diffusive convection regime. J. Phys. Oceanogr., 42, 840-854.

,-- , and,$- 2012 \mathrm{~b}$ : Simulations of a double-diffusive interface in the diffusive convection regime. J. Fluid Mech., 711, 411-436.

Descy, J.-P., F. Darchambeau, and M. Schmid, Eds., 2012: Lake Kivu: Limnology and Biogeochemistry of a Tropical Great Lake. Aquatic Ecology Series, Vol. 5, Springer, 190 pp.

Dorf, R. C., and R. H. Bishop, 2010: Modern Control Systems. 12th ed. Prentice Hall, 1104 pp.

Fofonoff, N. P., S. P. Hayes, and R. C. Millard Jr., 1974: W.H.O.I/ Brown CTD microprofiler: Methods of calibration and data handling. Woods Hole Oceanographic Institution Tech. Rep. WHOI-74-89, 64 pp.

Fozdar, F. M., G. J. Parker, and J. Imberger, 1985: Matching temperature and conductivity sensor response characteristics. J. Phys. Oceanogr., 15, 1557-1569.

Gregg, M. C., 1999: Uncertainties and limitations in measuring \& and X. J. Atmos. Oceanic Technol., 16, 1483-1490.

— thermistors. J. Geophys. Res., 85 (C5), 2779-2786.

Head, M., 1983: The use of miniature four-electrode conductivity probes for high resolution measurement of turbulent density or temperature variations in salt-stratified water flows. Ph.D. dissertation, University of California, San Diego, 211 pp.

Hill, K. D., 1987: Observations on the velocity scaling of thermistor dynamic response functions. Rev. Sci. Instrum., 58, 1235-1238. , and D. J. Woods, 1988: The dynamic response of the twoelectrode conductivity cell. IEEE J. Oceanic Eng., 13, 118123.

Johnson, G. C., J. M. Toole, and N. G. Larson, 2007: Sensor corrections for Sea-Bird SBE-41CP and SBE-41 CTDs. J. Atmos. Oceanic Technol., 24, 1117-1130.

Kelley, D. E., H. J. S. Fernando, A. E. Gargett, J. Tanny, and E. Özsoy, 2003: The diffusive regime of double-diffusive convection. Prog. Oceanogr., 56, 461-481.

Linden, P. F., and T. G. L. Shirtcliffe, 1978: The diffusive interface in double-diffusive convection. J. Fluid Mech., 87, 417-432.
Lueck, R. G., O. Hertzman, and T. Osborn, 1977: The spectral response of thermistors. Deep-Sea Res., 24, 951-970.

Meagher, T., A. Pederson, and M. C. Gregg, 1982: A low-noise conductivity microstructure instrument. IEEE Oceans 82, 283-290.

Mudge, T. D., and R. G. Lueck, 1994: Digital signal processing to enhance oceanographic observations. J. Atmos. Oceanic Technol., 11, 825-836.

Nash, J. D., and J. N. Moum, 1999: Estimating salinity variance dissipation rate from conductivity microstructure measurements. J. Atmos. Oceanic Technol., 16, 263-274.

$\longrightarrow$, and - 2002: Microstructure estimates of turbulent salinity flux and the dissipation spectrum of salinity. J. Phys. Oceanogr., 32, 2312-2333.

— D. R. Caldwell, M. J. Zelman, and J. N. Moum, 1999: A thermocouple probe for high-speed temperature measurement in the ocean. J. Atmos. Oceanic Technol., 16, 1474-1482.

Neal, V. T., S. Neshyba, and W. Denner, 1969: Thermal stratification in the Arctic Ocean. Science, 166, 373-374.

Newman, F., 1976: Temperature steps in Lake Kivu: A bottom heated saline lake. J. Phys. Oceanogr., 6, 157-163.

Proakis, J. G., and D. G. Manolakis, 1988: Introduction to Digital Signal Processing. MacMillan, 944 pp.

Schlichting, H., and K. Gersten, 2003: Boundary Layer Theory. 8th ed. Springer, $173 \mathrm{pp}$.

Schmid, M., M. Halbwachs, B. Wehrli, and A. Wüest, 2005: Weak mixing in Lake Kivu: New insights indicate increasing risk of uncontrolled gas eruption. Geochem. Geophys. Geosyst., 6, Q07009, doi:10.1029/2004GC000892.

_- M. Busbridge, and A. Wüest, 2010: Double-diffusive convection in Lake Kivu. Limnol. Oceanogr., 55, 225-238.

Schmitt, R. W., 1994: Double diffusion in oceanography. Annu. Rev. Fluid Mech., 26, 255-285.

—, R. C. Millard, J. M. Toole, and W. D. Wellwood, 2005: A double-diffusive interface tank for dynamic-response studies. J. Mar. Res., 63, 263-289.

Timmermans, M.-L., J. Toole, R. Krishfield, and P. Winsor, 2008: Ice-tethered profiler observations of the double-diffusive staircase in the Canada Basin thermocline. J. Geophys. Res., 113, C00A02, doi:10.1029/2008JC004829.

Vachon, P., and R. Lueck, 1984: A small combined temperatureconductivity probe. Proc. 1984 STD Conf. and Workshop, San Diego, California, Marine Technology Society San Diego Section and MTS Oceanic Instrumentation Committee, 126131.

Washburn, L., T. Duda, and D. C. Jacobs, 1996: Interpreting conductivity microstructure: Estimating the temperature variance dissipation rate. J. Atmos. Oceanic Technol., 13, 1166-1188.

Wolk, F., H. Yamazaki, L. Seuront, and R. G. Lueck, 2002: A new free-fall profiler for measuring biophysical microstructure. J. Atmos. Oceanic Technol., 19, 780-793.

Worster, M. G., 2004: Time-dependent fluxes across doublediffusive interfaces. J. Fluid Mech., 505, 287-307. 